\title{
EDITORIAL
}

For reprint orders, please contact: reprints@futuremedicine.com

\section{Challenges in the management of distal arm pain}

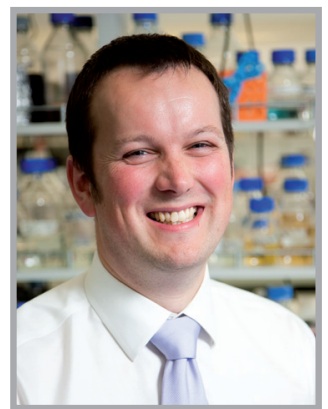

Gareth T Jones*

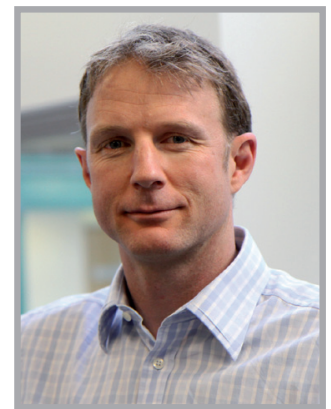

Peter J Heine ${ }^{1}$ "...there is a general lack of evidence regarding treatment for conditions of the distal arm, and no recommendations can be described as comprehensive."
Arm pain is a common cause of disability, demand for healthcare and lost work time. A recent population-based study found that approximately one in seven workingaged adults in England consulted their general practitioner with upper limb pain per annum [1]. Of these, $42 \%$ had pain in the distal arm and, at 12-month followup, nearly half were still in pain, with one in five reporting unremitting pain (never pain-free for as many as 7 consecutive days) [2].

Distal arm pain, defined as pain in the elbow, forearm, wrist or hand, may involve a number of specific pathologies, and also a variety of nonspecific syndromes. As with most musculoskeletal disorders, conditions of the distal arm have typically been categorized according to anatomical location and/or type of pathology, derived from clinical observation and practice. In many cases, validity and reliability for various diagnoses have not been established. Considerable uncertainty over classification and diagnosis has already been reported for upper limb disorders [3] and this is likely to be repeated in the distal arm. Hence, there is a variety of different diagnostic labels that may not be consistently applied between practitioners, nor have a valid pathophysiological basis. This is further confounded by the potential for referral of symptoms from other locations such as the thoracic/cervical spine or shoulder into the distal arm.

Apart from the more generic terms such as muscle strain, joint sprain or contusion, that may arise as a result of soft tissue trauma, other diagnostic labels for the distal arm pain include (but are not confined to) tendonitis, tenosynovitis, epicondylitis (in various guises including Golfers' or Tennis elbow), repetitive strain injury, bursitis, osteoarthritis, carpal tunnel syndrome and other nerve entrapment disorders. In general, the evidence regarding management of these conditions is poor [3] and most guidelines are based on expert recommendations rather than clinical trials. Typically, this is because few or no trials have been conducted, or the results of different trials have been contradictory, leaving clinicians with little to go on but their own experience, past practice and peer opinion. For example, the majority of the American College of Occupational and

\footnotetext{
"Typically ... few or no trials have been conducted, or the results of different trials have been contradictory, leaving clinicians with little to go on but their own experience, past practice and peer opinion.”
} 


\footnotetext{
"Clinical practitioners who scratch beneath the surface will be dismayed to find that most expert guidance is less substantial than they might hope...”
}

"...mechanical and psychosocial factors were found to be independently associated with an increase in the risk of pain onset in the distal arm."
Environmental Medicine's recommendations for management of elbow disorders [4] were based on consensus amongst a panel of convened experts, again derived from clinical experience, due to the lack of sufficient evidence from the literature for evidence-based recommendations. Of the 13 conditions and 24 modalities described, 74 recommendations were made. Only two conditions had been investigated by clinical trials in sufficient depth as to be able to formulate any recommendation based on their results. Epicondylitis had recommendations regarding eight modalities based on available evidence (two strong recommendations based on highquality evidence, four moderate recommendations based on intermediate evidence and two weak recommendations based on low-quality evidence), and ulnar nerve entrapment had two recommendations regarding two surgical techniques (both based on low-quality evidence). The other 64 recommendations concerning other conditions and treatment types were made without the benefit of any appropriate backing from the literature.

A review of the literature regarding management of conditions of the distal arm for the purposes of this article has come to similar conclusions. Electronic searches of health research databases, supplemented by evidence from Cochrane reviews and suitable meta-analyses, were conducted in order to locate clinical guidelines for the treatment of distal arm conditions. Only those which used the results of clinical trials and which rated the quality of the sources and the strength of evidence for their recommendations were included, considerably limiting the number available for review. In total, 44 different interventions used to treat 11 conditions of the distal arm were included. The strongest evidence for any modality was against the use of extracorporeal shock wave therapy [3-7] or laser therapy $[3,4,7,8]$ for disorders of the elbow. Although useful information, these modalities are not in widespread use and are unlikely to be considered mainstream treatment by most practitioners. Intermediate-to-strong evidence was reported for corticosteroid use in tenosynovitis [3], epicondylitis [4] and carpal tunnel syndrome [9-11], splinting for carpal tunnel syndrome [10,12] and NSAIDs for elbow disorders in general [4]. Of the rest, 32 intervention types (including various forms of exercise, massage, electrotherapy, manual therapy, heat/cold therapy and acupuncture) fell into the inevitable insufficient evidence' category preventing any meaningful recommendation regarding use or nonuse. This encompasses the most widely used interventions for most distal arm conditions.

In addition, although not confined to conditions of the distal arm, there is some support for various types of pain management/cognitive behavioral therapy programs, with or without some form of occupational or return to work component [3], which reflects the success of these types of interventions with conditions in other parts of the body (e.g., low back pain).

In summary, there is a general lack of evidence regarding treatment for conditions of the distal arm, and no recommendations can be described as comprehensive. Clinical practitioners who scratch beneath the surface will be dismayed to find that most expert guidance is less substantial than they might hope for various reasons, including:

- A lack of suitable trials on most conditions and/or modalities;

- Differences in definition of conditions;

- Differences in the use of trial methodology and modality;

- Recommendations based on very few studies;

- Differences in rating systems used by different expert panels.

So what do we know about the epidemiology of distal arm pain and can this inform management? In this area too, there have been few highquality studies. A large study of newly employed, predominantly male workers from 12 diverse occupational groups - from both sedentary and active environments - identified a number of risk factors for the onset of distal arm pain. Various mechanical occupational exposures were associated with an increase in the risk of future symptoms, including lifting and carrying heavy loads, working with hands above shoulder level and working with repetitive movements of wrists or arms. However, equally important - in fact, in some instances more so - were workplace psychosocial factors, including low levels of control, job dissatisfaction and perceiving one's job to be monotonous or boring. Furthermore, mechanical and psychosocial factors were found to be independently associated with an increase in the risk of pain onset in the distal arm [13]. Other authors have shown that beliefs about arm pain are important. Among those with distal arm 
pain, those who believe that physical activity may cause harm, that the pain would still be a problem in 3 months or that the pain is made worse by stress, are more likely to consult a general practitioner with their symptoms than other individuals [14]. Those with negative beliefs, and those with poorer self-rated general health and higher somatization scores, also experience a poorer prognosis.

Thus, in epidemiological terms, distal arm pain is very similar to low back pain: it is common and disabling, its etiology comprises both mechanical and psychosocial elements and beliefs are of demonstrable importance, not only in determining consultation but also in prognosis, as are aspects of general health. It also exhibits a similar lack of evidence for many of the diagnostic categories and treatment modalities commonly used. Many patients with distal arm pain are referred to physiotherapy where, while awaiting treatment, they are commonly advised to rest the 'injured' area. Indeed, in the UK, the online and telephone information and advice service provided by the National Health Service (NHS Direct) advises that: "If you are diagnosed with repetitive strain injury, or a work-related upper limb disorder it is likely that your general practitioner will initially advise you to stop doing the task or activity that is causing your symptoms." [101]. Such advice, however, lacks an evidence base. A recent search of the literature established that no randomized controlled trial has hitherto investigated the benefits of rest versus remaining active in the management of distal arm pain. Although the epidemiology is similar, the initial management of low back pain differs enormously. For many years, it too was managed by rest until trials demonstrated improved prognosis if patients remained mobile [15], an approach that has since been realized in community health campaigns $[16,17]$. With no strong reasons to believe that distal arm pain may benefit from a different management approach, one must assume that the differential advice reflects a wariness of employers and occupational physicians to allow employees with distal arm pain to continue with forceful or repetitive work activities for fear of possible litigation.

In summary, much of the initial advice commonly given to patients with conditions involving the distal arm is counter-intuitive. Perhaps, given what we know from epidemiology and the lack of evidence from clinical trials, it is time to look at approaches used in the management of other conditions such as lower back pain in an effort to improve care for patients with distal arm pain.

\section{Financial \& competing interests disclosure}

The authors have no relevant affliations or financial involvement with any organization or entity with a financial interest in or financial conflict with the subject matter or materials discussed in the manuscript. This includes employment, consultancies, honoraria, stock ownership or options, expert testimony, grants or patents received or pending, or royalties.

No writing assistance was utilized in the production of this manuscript.

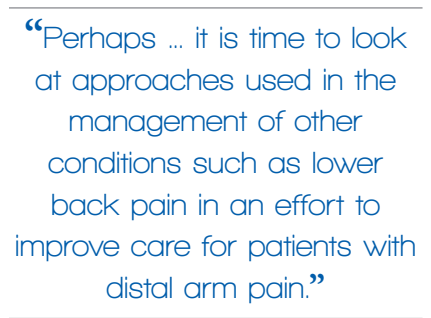

\section{References}

1 Palmer KT, Calnan M, Wainwright D et al. Upper limb pain in primary care: health beliefs, somatic distress, consulting and patient satisfaction. Fam. Pract. 23(6), 609-617 (2006).

2 Ryall C, Coggon D, Peveler R, Poole J, Palmer KT. A prospective cohort study of arm pain in primary care and physiotherapy - prognostic determinants. Rheumatology (Oxford) 46(3), 508-515 (2007).

3 Burton AK, Kendall NAS, Pearce BG, Birrell LN, Bainbridge LC. Management of Upper Limb Disorders and the Biopsychosocial Model (Research Report RR596). Health and Safety Executive (2008).

4 American College of Occupational and Environmental Medicine (ACOEM). Elbow Disorders. American College of
Occupational and Environmental Medicine (ACOEM), IL, USA (2007).

5 Buchbinder R, Green SE, Youd JM, Assendelft WJ, Barnsley L, Smidt N. Shock wave therapy for lateral elbow pain. Cochrane Database Syst. Rev. 4, CD003524 (2005).

6 Buchbinder R, Green SE, Struijs P. Tennis elbow. Clin. Evid. 2008, 1117 (2008).

7 Bisset L, Paungmali A, Vicenzino B, Beller E. A systematic review and meta-analysis of clinical trials on physical interventions for lateral epicondylalgia. $\mathrm{Br}$. J. Sports Med. 39(7), 411-422 (2005).

8 Trudel D, Duley J, Zastrow I, Kerr EW, Davidson R, MacDermid JC. Rehabilitation for patients with lateral epicondylitis: a systematic review. J. Hand Ther. 17(2), 243-266 (2004).

9 O'Connor D, Marshall S, Massy-Westropp N. Non-surgical treatment (other than steroid injection) for carpal tunnel syndrome. Cochrane Database Syst. Rev. 1, CD003219 (2003).

10 Ashworth NL. Carpal tunnel syndrome. Clin. Evid. 2010, 1114 (2010).

11 Marshall S, Tardif G, Ashworth N. Local corticosteroid injection for carpal tunnel syndrome. Cochrane Database Syst. Rev. 2, CD001554 (2007).

12 American Academy of Orthopaedic Surgeons. Clinical Practice Guideline on the Treatment of Carpal Tunnel Syndrome. American Academy of Orthopaedic Surgeons, IL, USA (2008).

13 Nahit ES, Taylor S, Hunt IM, Silman AJ, Macfarlane GJ. Predicting the onset of forearm pain: a prospective study across 12 occupational groups. Arthritis Rheum. 49(4), 519-525 (2003).

14 Palmer KT, Reading I, Linaker C, Calnan M, Coggon D. Population-based 


\section{EDITORIAL Jones \& Heine}

cohort study of incident and persistent arm pain: role of mental health, self-rated health and health beliefs. Pain 136(1-2), 30-37 (2008).

15 Waddell G, Feder G, Lewis M. Systematic review of bed rest and advice to stay active for acute low back pain. Br. J. Gen. Pract. 47, 647-652 (1997).
16

Buchbinder R, Jolley D, Wyatt M. Population based intervention to change back pain beliefs and disability: three part evaluation. $B M J$ 322(7301), 1516-1520 (2001).

17 Buchbinder R, Jolley D. Population based intervention to change back pain beliefs: three year follow up population survey. BMJ 328(7435), 321 (2004).

\section{- Website}

101 NHS. Repetitive Strain Injury - Treatment. www.nhs.uk/Conditions/Repetitive-straininjury/Pages/Treatment.aspx 